\title{
Demonstration of Long-Lived High-Power Optical Waveguides in Air
}

\author{
N. Jhajj, E. W. Rosenthal, R. Birnbaum, J. K. Wahlstrand, and H. M. Milchberg** \\ Institute for Research in Electronics and Applied Physics, University of Maryland, \\ College Park, Maryland 20742, USA
}

(Received 7 November 2013; revised manuscript received 8 December 2013; published 26 February 2014)

\begin{abstract}
We demonstrate that femtosecond filaments can set up an extended and robust thermal waveguide structure in air with a lifetime of several milliseconds, making possible the very-long-range guiding and distant projection of high-energy laser pulses and high-average power beams. As a proof of principle, we demonstrate guiding of 110-mJ, 7-ns, 532-nm pulses with 90\% throughput over 15 Rayleigh lengths in a 70-cm-long air waveguide generated by the long time-scale thermal relaxation of an array of femtosecond filaments. The guided pulse was limited only by our available laser energy. In general, these waveguides should be robust against the effects of thermal blooming of extremely high-average-power laser beams.
\end{abstract}

DOI: 10.1103/PhysRevX.4.011027

\section{INTRODUCTION}

Long-range filamentation of intense femtosecond laser pulses in gases is an area of increasing interest, as it combines exciting potential applications with fundamental nonlinear optical physics [1,2]. An intense pulse propagating in a transparent medium induces a positive nonlinear correction to the refractive index that copropagates with the pulse as a self-lens. Once the laser-pulse peak power exceeds a critical value, typically $P>P_{\mathrm{cr}} \sim 5-10 \mathrm{GW}$ in gases [1,3], the self-induced lens overcomes diffraction and focuses the beam, leading to plasma generation and beam defocusing when the gas ionization intensity threshold is exceeded. The dynamic interplay between self-focusing and defocusing leads to self-sustained propagation of a tightly radially confined high-intensity region accompanied by plasma of diameter $<100 \mu \mathrm{m}$ [4] over distances greatly exceeding the optical Rayleigh range. Filaments can extend from millimeters to hundreds of meters, depending on the medium and laser parameters [1]. Among the applications of filaments are remote sensing [5], $\mathrm{THz}$ generation [6,7], spectral broadening and compression of ultrashort laser pulses [8,9], and channeling of electrical discharges [10]. Despite these applications, it remains a significant limitation that femtosecond filamentation cannot deliver high average power over long distances in a single tight spatial mode. This is due to the fact that for laser pulses with $P \sim$ several $P_{\mathrm{cr}}$, the beam will collapse into multiple filaments [11] with

\footnotetext{
*Corresponding author. milch@umd.edu

Published by the American Physical Society under the terms of the Creative Commons Attribution 3.0 License. Further distribution of this work must maintain attribution to the author(s) and the published article's title, journal citation, and DOI.
}

Subject Areas: Interdisciplinary Physics, Optics, Photonics

shot-to-shot variation in their transverse location. For $P_{\mathrm{cr}} \sim 5-10 \mathrm{GW}$, this means that single-filament formation requires pulses of order $1 \mathrm{~mJ}$. For a $1-\mathrm{kHz}$ pulse repetition rate laser, this represents only $1 \mathrm{~W}$ of average power.

Here, we demonstrate a method employing filaments that can easily supersede this limitation by setting up a robust, long-range optical guiding structure lasting milliseconds. It opens the possibility for optical guiding of megawatt levels of average power over long distances in the atmosphere. The guiding structures demonstrated here have substantial potential for directed energy applications [12].

The generation of long-lived thermal guiding structures in air using filament arrays also has the potential to enhance other photonics applications in the atmosphere. For instance, they could be used to concentrate heater beams for remote atmospheric lasing schemes [13] or for inducing characteristic emission for standoff detection of chemical compounds. Many remote detection applications rely on the collection of fluorescence $[5,14,15]$. For these remotesensing schemes, where detection over large distances may be desired, very little of the isotropically emitted fluorescence reaches the detector at a distance. The long-lived guiding structures demonstrated here could be used as an effective collection lens, enhancing the signal. They may also find use in atmospheric laser communication [16]. In addition, they might be used to enhance and control the propagation of an injected ultrashort filamenting pulse [17], similar to what has been done with a permanent refractive index structure in glass [18] and recently with the plasma from an array of filaments in air [19].

We note that there has been much recent work on using the refractive index of the plasma generated by an array of filaments to form guides for microwaves [20,21] and nanosecond optical pulses [22]. We emphasize that the guiding we demonstrate here does not use the optical 


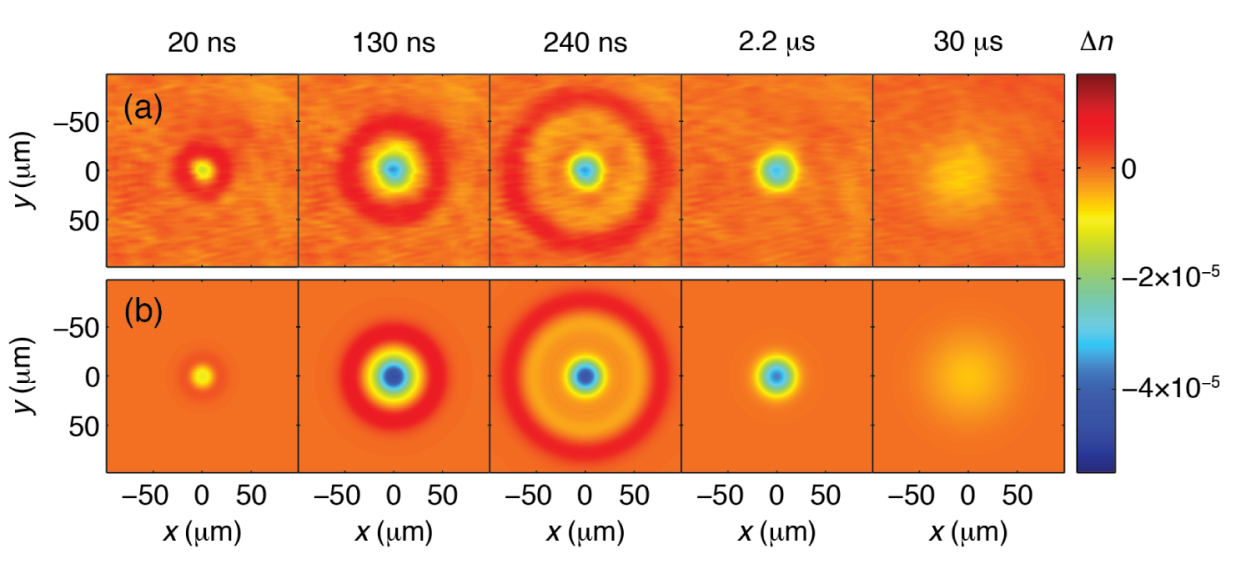

FIG. 1. Gas dynamics following a single filament in air. (a) Interferometric measurement of the refractive index change following a short pulse as a function of the time delay of the probe pulse. (b) Hydrodynamic simulation, assuming a 60- $\mu \mathrm{m}$ FWHM Gaussian heat source of peak initial density $32 \mathrm{~mJ} / \mathrm{cm}^{3}$.

response of the plasma-rather, it uses the $10^{6}$-times-longerduration hydrodynamic response of the gas after heating by the filaments.

\section{RESULTS AND DISCUSSION}

\section{A. Gas hydrodynamics initiated by femtosecond filaments}

Recently we found that a femtosecond filament, starting at an electron temperature and density of a few $\mathrm{eV}$ and a few times $10^{16} \mathrm{~cm}^{-3}$ [4], acts as a thermal source to generate long-lived gas-density hole structures that can last milliseconds and dissipate by thermal diffusion [23]. In air, additional heating can occur from molecular excitation [23], with peak deposited energy density in the plasma and molecules of as much as $\sim 100 \mathrm{~mJ} / \mathrm{cm}^{3}$. Our earlier gasdensity measurements [23] were limited to $\sim 40-\mu s$ resolution, so the early-time dynamics on the nanosecond time scale had been simulated but not directly measured. These structures are initiated as the filament plasma recombines to a neutral gas on a 10 -ns time scale and the molecular excitation (if applicable) thermalizes. Owing to the finite thermal conductivity of the gas, the initial energy invested in the filament is still contained in a small radial zone, but it is repartitioned into the translational and rotational degrees of freedom of the neutral gas. The result is an extended and narrow high-pressure region at temperatures up to a few hundred $\mathrm{K}$ above ambient. In air, this pressure source launches a radial sound wave $\sim 100 \mathrm{~ns}$ after the filament is formed. By $\sim 1 \mu s$, the gas reaches pressure equilibrium with an elevated temperature and reduced gas density in the volume originally occupied by the filament, after which the "density hole" decays by thermal diffusion on a fewmillisecond time scale [23].

The full dynamics are now clearly seen in Fig. 1(a), which presents new, higher time-resolution measurements of the 2D density hole evolution (expressed as an air refractive index shift) of a short air filament from nanoseconds through microseconds after filament formation. A 1D radial fluid code simulation, described in Appendix A, is shown in Fig. 1(b) for comparison, and the results are in excellent agreement with the measurements. The experimental results verify that the density hole first deepens over tens of nanoseconds and launches a sound wave that propagates beyond the $\sim 200-\mu \mathrm{m}$ frame by $\sim 300 \mathrm{~ns}$. By $\sim 1-2 \mu \mathrm{s}$, pressure equilibrium is reached and the hole decays by thermal diffusion out to millisecond time scales.

The gas-density measurements were performed using the setup shown in Fig. 2(a) and described in Appendix B. A 532-nm, 7-ns laser pulse was used either as a longitudinal interferometric probe or as an injection source for optical guiding. The transverse gas-density profiles shown in Fig. 1(a) were obtained using the 532-nm pulse as an interferometric probe of a single short $\sim 2-\mathrm{mm}$ filament. The short filament length is essential for minimizing refractive distortion of the interferometric probe pulse [24].

We note that at no probe delay for a single filament do we see an on-axis refractive index enhancement, as claimed in Ref. [25], that might explain their recent report of guiding [25], an issue further discussed in Ref. [26]. At the longer delays of tens of microseconds and beyond, the thermal gas-density hole acts as a negative lens, as seen in our earlier experiments [23].

\section{B. Multifilament-induced guiding structure}

Although a single filament results in a beam-defocusing gas-density hole, a question arises as to whether a guiding structure can be built using the judicious placement of more than one filament. We tested this idea with a four-lobed focal beam structure using two orthogonal "half-pellicles." As seen in Fig. 2(a), the pellicles are oriented to phase shift the laser electric field as shown in each near-field beam quadrant. Below the filamentation threshold, the resulting focused beam at its waist has a four-lobed intensity profile as shown, corresponding to a Hermite-Gaussian TEM 11 mode, where the electric fields in adjacent lobes are $\pi$ phase 
(a)

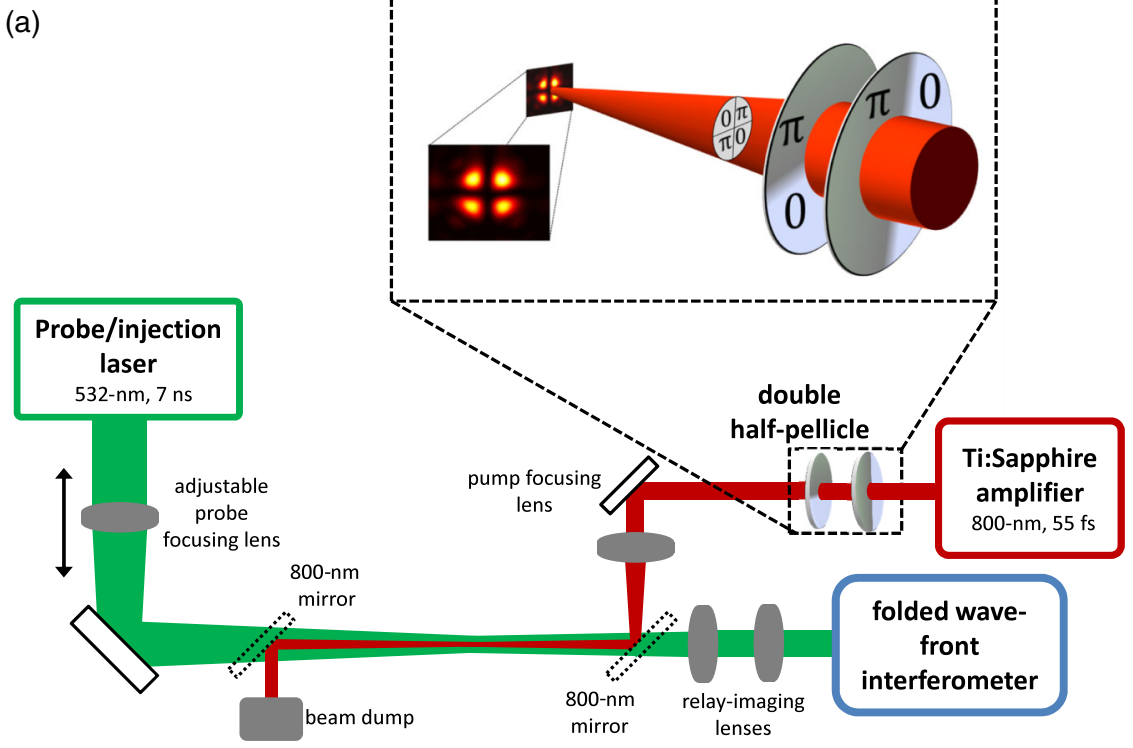

(b)
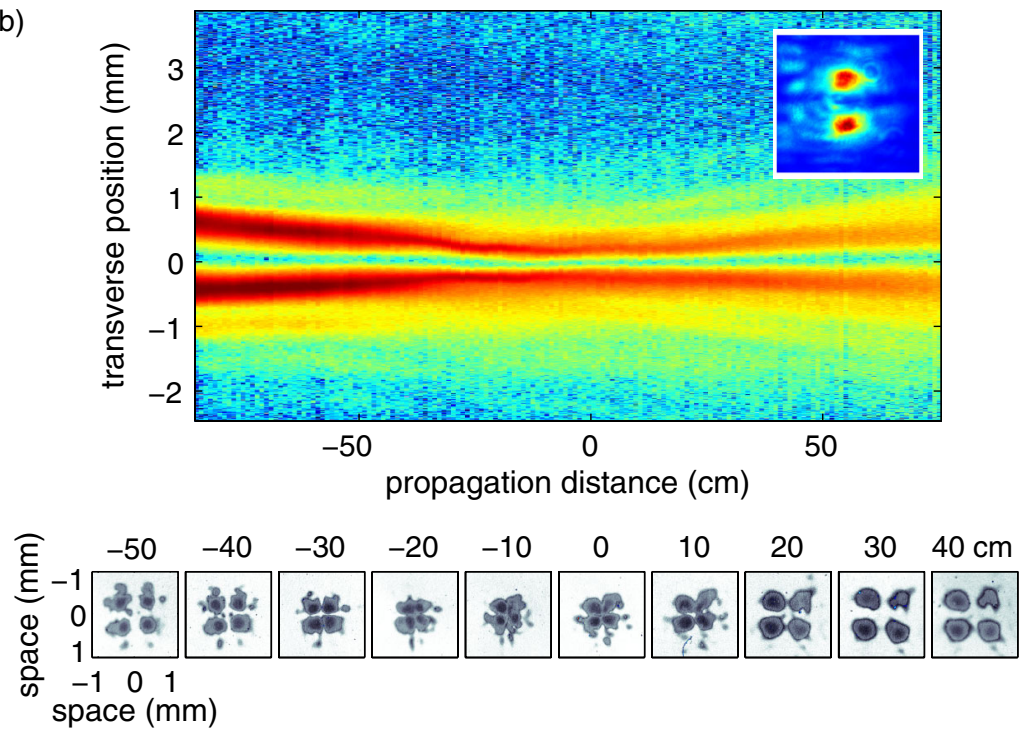

FIG. 2. Generation of a filament array using half-pellicles. (a) A 55-fs, 800-nm, 10-Hz pulsed laser is used to generate an array of four filaments. A pulse propagates through two orthogonal half-pellicles, inducing $\pi$ phase shifts on neighboring quadrants of the beam, which is then focused to produce a four-filament with a TEM $_{11}$ mode (actual low-intensity image shown). A 7-ns, 532-nm 10-Hz pulsed laser counterpropagates through the filament and is imaged either directly onto a CCD for guiding experiments or through a folded wavefront interferometer and onto a CCD for interferometry. (b) Rayleigh scattering as a function of $z$ with a bifilament produced by a single half-pellicle (the bifilament far-field mode is shown in the inset). The bottom row shows $z$-dependent burn patterns produced by a four-filament produced by two orthogonal half-pellicles.

shifted with respect to each other. Above the threshold, the lobes collapse into filaments whose optical cores still maintain this phase relationship, and thus four parallel filaments are formed. As a demonstration of this, the top panel of Fig. 2(b) shows an image of the Rayleigh side scattering at $800 \mathrm{~nm}$ from a two-lobed filament produced by a single half-pellicle, indicating that the $\pi$ phase shift is preserved along the full length of the filament. This image was obtained by concatenating multiple images from a low-noise CCD camera translated on a rail parallel to the filament. The images were taken through an 800 -nm interference filter. The bottom panel shows burn patterns taken at multiple locations along the path of a $\sim 70$-cm-long fourlobe filament used later. For the $70-\mathrm{cm}$ four-filament, the filament core spacing is roughly constant at $\sim 300 \mu \mathrm{m}$ over a $L \sim 50 \mathrm{~cm}$ region with divergence to $\sim 1 \mathrm{~mm}$ at the ends.

The effect of a four-filament structure on the gas dynamics is shown in Fig. 3, a sequence of gas-density profiles measured for a short $\sim 2$-mm filament (produced at $f / 35$ ) to minimize refractive distortion of the probe beam. 


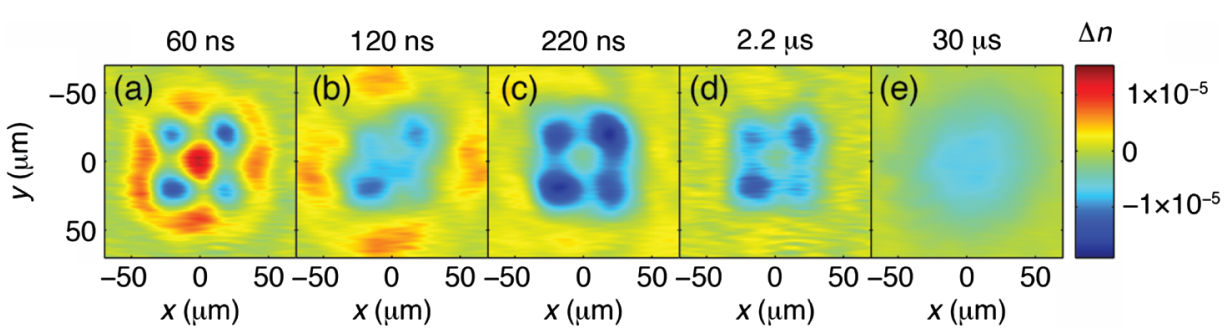

FIG. 3. Interferometric measurement of the air density evolution induced by a four-filament. (a) The acoustic waves generated by each filament cross in the middle, generating a positive index shift, producing the acoustic guide. (b) The acoustic waves propagate outward, leaving behind a density depression at the location of each filament. (c) The density depressions produce the thermal guide, with a higher central density surrounded by a moat of lower density. (d, e) The density depressions gradually fill in as the thermal energy dissipates. A movie of the four-filament-induced gas evolution is provided in Ref. [27].

The peak intensity was $<10^{14} \mathrm{~W} / \mathrm{cm}^{2}$, typical of the refraction-limited intensity in more extended filaments, so we expect these images to be descriptive of the gas dynamics inside much longer filaments. Inspection of the density profiles shows that there are two regimes in the gas dynamical evolution which are promising for supporting the guiding of a separate injected laser pulse. A shorterduration, more-transient acoustic regime occurs when the sound waves originating from each of the four filaments superpose at the array's geometric center, as seen in panel (a) of Fig. 3, causing a local density enhancement of approximately a factor of 2 larger than the sound-wave amplitude, peaking $\sim 80 \mathrm{~ns}$ after filament initiation and lasting approximately $\sim 50 \mathrm{~ns}$. A far-longer-lasting and significantly more-robust profile suitable for guiding is achieved tens of microseconds later, well after the sound waves have propagated far from the filaments. In this thermal regime, the gas is in pressure equilibrium [23]. As seen in panels (c) and (d) of Fig. 3, thermal diffusion has smoothed the profile in such a way that the gas at the center is surrounded by a "moat" of lower density. The central density can be very slightly lower than the far background because its temperature is slightly elevated, yet it is still higher than the surrounding moat. The lifetime of this structure can be several milliseconds. In both the acoustic and thermal cases, the diameter of the air-waveguide "core" is approximately half the filament lobe spacing. A movie of the four-filamentinduced gas evolution is provided in Ref. [27].

\section{Fiber analysis of air waveguides}

Having identified two potential regimes for optical guiding, a short-duration acoustic regime, and a muchlonger-duration thermal regime, it is first worth assessing the coupling and guiding conditions for an injected pulse. We apply the fiber parameter $V$ for a step index guide [28] to the air waveguide, $V=(2 \pi a / \lambda)\left(n_{\mathrm{co}}{ }^{2}-n_{\mathrm{cl}}{ }^{2}\right)^{1 / 2}$ $\sim(2 \sqrt{2} \pi a / \lambda)\left(\delta n_{\mathrm{co}}-\delta n_{\mathrm{cl}}\right)^{1 / 2}$, where the effective core and cladding regions have refractive indices $n_{\mathrm{co}, \mathrm{cl}}=n_{0}+$ $\delta n_{\mathrm{co}, \mathrm{cl}}$, where $n_{0}$ is the unperturbed background air index $\left(n_{0}-1=2.77 \times 10^{-4}\right.$ at room temperature and pressure [29]), $\delta n_{\mathrm{co}}$ and $\delta n_{\mathrm{cl}}$ are the (small) index shifts from background at the core and cladding, and the core diameter is $2 a$, taken conservatively at the tightest spacing of the filament array. The numerical aperture of the guide is $N A=\lambda V /(2 \pi a)$. Because accurate density profile measurements are restricted to short filaments, we use the results of Fig. 3 and apply them to much longer and widerlobe-separated filaments that are inaccessible to longitudinal interferometry owing to probe refraction. As typical filament core intensities are restricted by refraction ("intensity clamping") to levels $<10^{14} \mathrm{~W} / \mathrm{cm}^{2}$ [1], we expect that the measurements of Fig. 3 apply reasonably well to longer filaments and different lobe spacings. For the acoustic guide, we used a filamenting beam with lobe spacing of $150 \mu \mathrm{m}$, so $2 a \sim 75 \mu \mathrm{m}$. Using $\delta n_{\text {co }} /\left(n_{0}-1\right) \sim$ 0.05 and $\delta n_{\mathrm{cl}} /\left(n_{0}-1\right) \sim-0.02$ from Fig. 3 then gives $V \sim 2.8(>2.405)$ and $N A \sim 6.3 \times 10^{-3}$, indicating a nearsingle mode guide with an optimum coupling $f$-number of $f / \#=0.5 / N A \sim 80$. For the long thermal guide, we used a filamenting beam with lobe spacing of $\sim 300 \mu \mathrm{m}$, so $2 a \sim 150 \mu \mathrm{m}$. From Fig. 3, the core index shift is $\delta n_{\mathrm{co}} \sim$ 0 and the cladding shift is the index decrement at the moat, $\delta n_{\mathrm{cl}} /\left(n_{0}-1\right) \sim-0.02$, giving $V \sim 2.9$, corresponding to a near-single mode guide with $N A \sim 3.2 \times 10^{-3}$, corresponding to $f / \# \sim 160$.

\section{Injection and guiding experiments}

The experimental setup is shown in Fig. 2(a). An endmode image from injection and guiding of a low-energy $\lambda=532-\mathrm{nm}$ pulse in the acoustic waveguide produced from a 10-cm-long four-filament is shown in Fig. 4. In order to differentiate between guiding and the propagation of the unguided beam through the fully dissipated guide at later times $(>2 \mathrm{~ms})$, we define the guiding efficiency as $\left(E_{g}-E_{\mathrm{ug}}\right) /\left(E_{\mathrm{tot}}-E_{\mathrm{ug}}\right)$, where $E_{g}$ is the guided energy within the central mode, $E_{\text {tot }}$ is the total beam energy, and $E_{\mathrm{ug}}$ is the fraction of energy of the unguided mode occupying the same transverse area as the guided mode.

The best coupling in the acoustic regime occurred at an injection delay of $\sim 200 \mathrm{~ns}$ and $f / \#>100$, with a peak guided efficiency of $13 \%$, although the guides were not stable on a shot-to-shot basis. Efficient guiding in the 

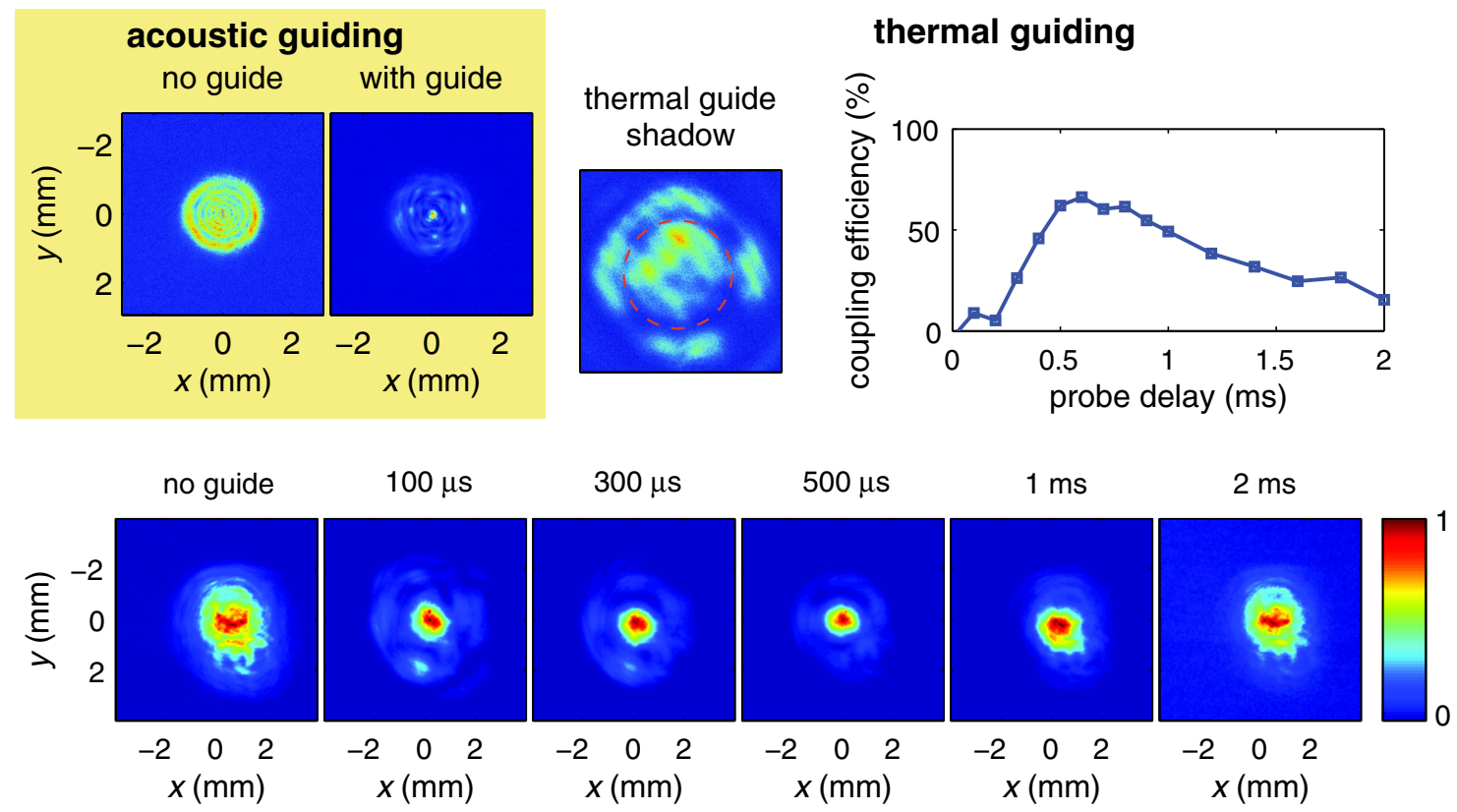

FIG. 4. Demonstration of guiding of 7-ns, $\lambda=532-\mathrm{nm}$ pulses in 70-cm-long acoustic and thermal air waveguides produced by a four-filament. The panel in the upper left shows the probe beam, which is imaged after the filamentation region, with and without the filament. The time delay of the probe was $200 \mathrm{~ns}$, which is in the acoustic guiding regime. The effect of the thermal waveguide, the shadow of which can be seen in the image in the top center (with a red dashed circle showing the position of the lower-density moat), is shown in the bottom row, where the probe beam is imaged after the exit location of the air waveguide with and without the filamenting beam. The coupling efficiency vs injected pulse delay is shown in the upper right. Peak energy guided was $110 \mathrm{~mJ}$.

acoustic regime takes place over an injection delay interval of only $\sim 100 \mathrm{~ns}$, consistent with the time for a sound wave to cross the waveguide core region, $a / c_{s} \sim 100 \mathrm{~ns}$, where $2 a=75 \mu \mathrm{m}$ and $c_{s} \sim 3.4 \times 10^{4} \mathrm{~cm} / \mathrm{s}$ is the air sound speed [30]. We found that for longer filaments with wider lobe spacings, the acoustic guides were even less stable. Unless the four-filament lobes were well balanced in energy and transverse position, the sound-wave superposition would not form a well-defined air-waveguide core. This is why a shorter $10-\mathrm{cm}$ filament was used for the acoustic-guide experiment. While the acoustic superposition guide is a promising approach, future experiments will need filaments generated by very well-balanced multilobe beam profiles, an example of which is seen in Ref. [26].

By comparison, the thermal guides were far more robust, stable, and long-lived. Results from the thermal guide produced by a 70-cm-long four-filament are also shown in Fig. 4, where optimal coupling was found for $f / \#=200$, in rough agreement with the earlier fiberbased estimate. An out-of-focus end-mode image (not to scale) is shown to verify the presence of the thermal guide's lower-density moat. Here, we note that owing to the much greater lobe spacing of its long four-filament, the thermal guide of Fig. 4 lasts much longer (milliseconds) than that from the short four-filament of Fig. 3 $(\sim 10 \mu s)$. Guided output modes as a function of injection delay are shown imaged from a plane past the end of the guide, in order to minimize guide distortion of the imaging. These mode sizes are larger than upstream in the guide where four-filament lobe spacing is tighter, but where we are unable to image reliably. We injected up to $110 \mathrm{~mJ}$ of 532-nm light, the maximum output of our laser, with 90\%-energy throughput in a single guided mode. This corresponds to a peak guiding efficiency of $70 \%$. Guiding efficiency vs injected pulse delay is plotted in Fig. 4. As seen in that plot, peak guiding occurs at $\sim 600 \mu \mathrm{s}$ and persists out to $\sim 2 \mathrm{~ms}$, where the guiding efficiency drops to $\sim 15 \%$. Based on the guide core diameter of $2 a \sim 150 \mu \mathrm{m}$ and the portion of the filament length with constant lobe spacing, $L \sim 50 \mathrm{~cm}$, the guided beam propagates approximately $L \lambda /\left(\pi a^{2}\right) \sim 15$ Rayleigh ranges. A movie of the thermal waveguide output beam, during real-time rastering (at $10 \mathrm{~Hz}$ ) of the injected beam across the guide entrance, is shown in Ref. [27].

\section{E. Simulations of waveguide development and guiding}

Owing to the linearity of the heat-flow problem, the evolution of the four-filament-induced density structure in the thermal regime can be calculated by finding the solution $T(x, y, t)$ to the 2D heat-flow equation, $\partial T / \partial t=\alpha \nabla^{2} T$, for a single filament source located at $(x, y)=(0,0)$ and then forming $T_{4}(x, y, t)=\sum_{j=1}^{4} T\left(x-x_{j}, y-y_{j}, t\right)$, where $\left(x_{j}, y_{j}\right)$ are the thermal source locations in the fourfilament. Here, $\alpha=\kappa / c_{p}$, where $\kappa$ and $c_{p}$ are the thermal conductivity and specific heat capacity of air. To an excellent approximation, as shown in Ref. [23], $T(x, y, t)$ is Gaussian in space. Invoking pressure balance, the $2 \mathrm{D}$ density evolution is then given by 


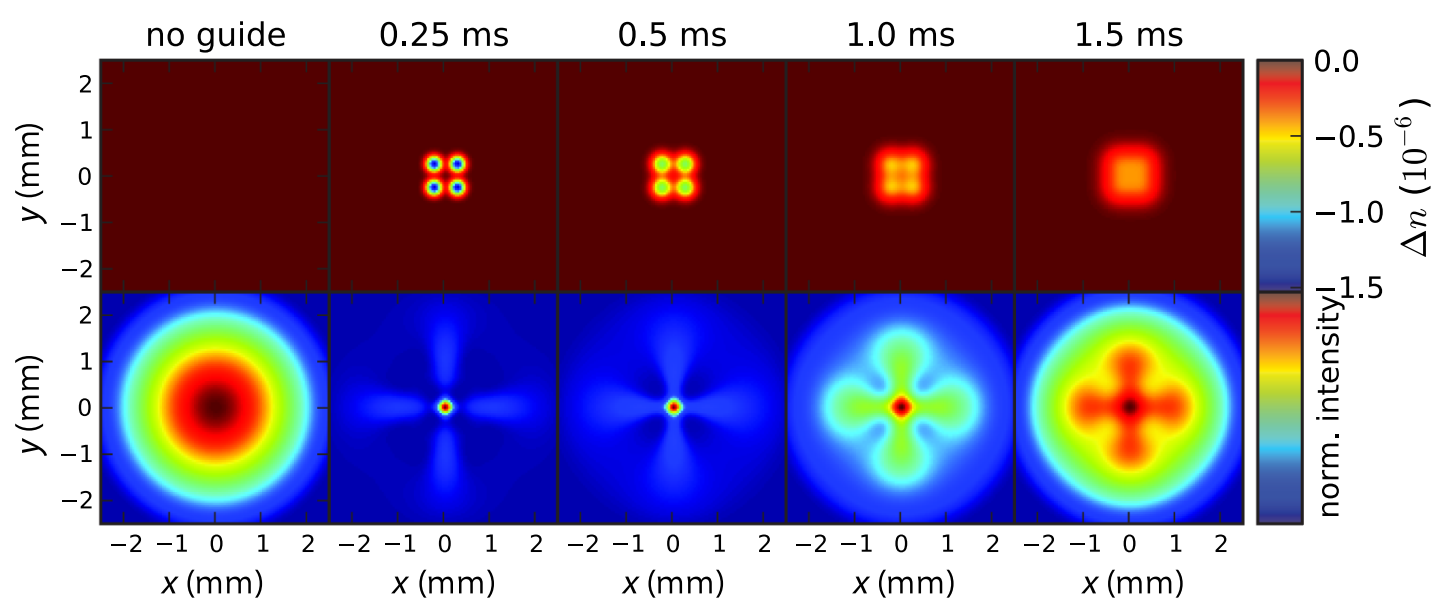

FIG. 5. Simulation of the evolution of and guiding in a thermal air waveguide. The top row shows the index of refraction shift produced by the four-filament-induced temperature profile as a function of time. The bottom row shows a BPM simulation of the guided laser-beam profile at the end of a $70-\mathrm{cm}$ waveguide produced by the four-filament-induced refractive index change.

$$
\begin{aligned}
\Delta N_{4}(r, t)= & -N_{b} \frac{\Delta T_{0}}{T_{b}}\left(\frac{R_{0}^{2}}{R_{0}^{2}+4 \alpha t}\right) \\
& \times \sum_{j=1}^{4} \exp \left(\frac{-\left(x-x_{j}\right)^{2}-\left(y-y_{j}\right)^{2}}{R_{0}^{2}+4 \alpha t}\right),
\end{aligned}
$$

where $R_{0}$ is the initial $1 / e$ radius of the temperature profile of a single filament and $\Delta T_{0}$ is its peak value above $T_{b}$, the background (room) temperature. Using $R_{0}=50 \mu \mathrm{m}$, $\Delta T_{0}=15 \mathrm{~K}, \alpha=0.21 \mathrm{~cm}^{2} / \mathrm{s}$ for air [23], and source locations separated by $500 \mu \mathrm{m}$, approximating our $70-\mathrm{cm}$ four-filament, gives the sequence of gas-density plots shown in the upper panels of Fig. 5, clearly illustrating the development and persistence of the guiding structure over milliseconds.

The propagation of the 532-nm beam in the waveguide was simulated in the paraxial approximation using the beam propagation method (BPM) [31]. The calculated intensity at the output of the waveguide is shown in the lower panels of Fig. 5. At early delays $<100 \mu \mathrm{s}$, characteristics of a multimode waveguide are observed in the simulation, including mode beating. At later times, as the refractive index contrast decreases, the propagation is smoother, indicating singlemode behavior, consistent with the estimates in Sec. C using the fiber parameter. The simulation is in reasonable agreement with the experimental results. Axial nonuniformity in the waveguiding structure could explain the absence of fourfold symmetry in the experimental data, whereas it is pronounced in the simulations.

\section{CONCLUSIONS}

We have demonstrated the generation of very-long-lived and robust optical waveguides in air, their extent limited only by the propagation distance of the initiating femtosecond filament array and the axial uniformity of its energy deposition. Assuming a sufficiently uniform filament, this is ultimately determined by the femtosecond pulse energy absorbed to heat the gas. Based on a single filament diameter of $\sim 100 \mu \mathrm{m}$, an electron density of $\sim 3 \times 10^{16} \mathrm{~cm}^{-3}$ [4], ionization energy of $\sim 10 \mathrm{eV}$ per electron, and $\sim 5 \mathrm{meV}$ of heating per air molecule [23], approximately $0.5 \mathrm{~mJ}$ is needed per meter of each filament. With a femtosecond laser system of a few hundred millijoules pulse energy, waveguides hundreds of meters long are possible.

What is the optical-power-carrying capacity of these guides? For high peak power pulses, the peak guided intensity, $I_{p}\left(W / \mathrm{cm}^{2}\right)$, is limited by self-focusing in the waveguide core. For $\lambda=1 \mu \mathrm{m}$ guided light, the approximate B-integral condition is $I_{p} L_{g}<\sim 2 \times 10^{14} \mathrm{~W} / \mathrm{cm}$ where $L_{g}$ is the guide length $(\mathrm{cm})$ and the nonlinear index of air is obtained from Ref. [3]. For our $\sim 100 \mathrm{~cm}$ waveguide, this gives $I_{p}<\sim 2 \times 10^{12} \mathrm{~W} / \mathrm{cm}^{2}$, corresponding to a pulse energy of $\sim 4 \mathrm{~J}$ for the $150-\mu \mathrm{m}$ diameter core, assuming a $10 \mathrm{~ns}$ pulse. At higher repetition rates $(>1 \mathrm{kHz})$ and over longer ranges (hundreds of meters), heating from non-linear absorption mechanisms such as stimulated Raman scattering (SRS) may also become relevant for guided energetic nanosecond pulses. However, the real utility of these air waveguides, in the thermal formation regime, derives from their extremely long millisecond-scale lifetime. This opens the possibility of guiding very-high-average powers that are well below the self-focusing or SRS thresholds.

We now consider the robustness of our filament-induced waveguides to thermal blooming [12,32] from molecular and aerosol absorption in the atmosphere. For thermal blooming, we consider the deposited laser energy that can raise the local gas temperature by a fraction $\eta$ of ambient, $P_{\mathrm{g}} \Delta t / A=1.5 \eta \alpha^{-1} p$, where $P_{\mathrm{g}}$ is the guided laser power, $\Delta t$ is the pulse duration, $\alpha$ is the absorption coefficient, $A$ is the waveguide core cross-sectional area, and $p$ is the ambient pressure. Thermal blooming competes with guiding when $\eta$ is approximately equal to the relative gas-density difference between the core and cladding. In 
our measurements of the thermal air waveguide, the typical index (and density) difference between the core and cladding is of the order of $\sim 2 \%$ at millisecond time scales. Taking $\eta=0.02, p=1 \mathrm{~atm}$, and $\alpha=2 \times 10^{-8} \mathrm{~cm}^{-1}$ [12] gives $P_{\mathrm{g}} \Delta t / A<\sim 1.5 \times 10^{5} \mathrm{~J} / \mathrm{cm}^{2}$ as the energy flux limit for thermal blooming. For example, for a $1.5-\mathrm{mm}$ diameter airwaveguide core formed from an azimuthal array of filaments [26], the limiting energy is $P_{\mathrm{g}} \Delta t \sim 2.7 \mathrm{~kJ}$. Note that we use a conservative value for $\alpha$ at $\lambda \sim 1 \mu \mathrm{m}$ that includes both molecular and aerosol absorption for maritime environments [12], which contain significantly higher aerosol concentrations than dry air. If a high-power laser is pulsed for $\Delta t$ $\sim 2 \mathrm{~ms}$, consistent with the lifetime of our $10-\mathrm{Hz}$-generated thermal waveguides, the peak average power can be $1.3 \mathrm{MW}$. It is possible that in such environments, air heating by the filament array itself could help dissipate the aerosols before the high-power beam is injected, further raising the thermal blooming threshold and also reducing aerosol scattering. An air waveguide even more robust against thermal blooming and capable of quasicontinuous operation may be possible using kHz-repetition-rate filamenting laser pulses. We have already shown that the cumulative effect of filamenting pulses arriving faster than the density hole can dissipate leads to steady-state hole depths of order $\sim 10 \%$ [23].

\section{ACKNOWLEDGMENTS}

N. Jhajj and E. W. Rosenthal contributed equally to this work. The authors thank P. Sprangle, J. Palastro, S. Yoon, and S. Zahedpour for useful discussions. This work is supported by the Air Force Office of Scientific Research, the National Science Foundation, the Department of Energy, and the Office of Naval Research. H. M. M. thanks his father for non-technical discussions.

\section{APPENDIX A: SIMULATION OF FILAMENT-INDUCED GAS DYNAMICS}

The simulations of the gas hydrodynamic evolution is performed in cylindrical geometry using a one-dimensional Lagrangian one-fluid hydrocode, in which the conservation equations for mass, momentum, and energy, $\partial \xi_{i} / \partial t+$ $\nabla \cdot\left(\xi_{i} \mathbf{v}+\phi_{i}\right)=S_{i}$, were solved numerically. For the mass equation, $\xi_{1}=\rho$ and $\phi_{1}=0$; for the momentum equation, $\xi_{2}=\rho \mathbf{v}$ and $\phi_{2}=P \overleftrightarrow{\mathbf{I}}$ (where $\overleftrightarrow{\mathbf{I}}$ is the unit tensor); and for the energy equation, $\xi_{3}=\varepsilon+1 / 2 \rho v^{2}$ and $\phi_{3}=P \mathbf{v}+\mathbf{q}$. Here, $\xi$ is the volume density of the conserved quantity, $\phi$ is the flux of that quantity, and $S$ refers to sources or sinks, while $\rho$ is the mass density, $\varepsilon$ is the fluid internal energy density, $\mathbf{v}$ is the fluid velocity ( $v$ is its magnitude), $P$ is the gas pressure, and $\mathbf{q}=-\kappa \nabla T$ is the heat flux, where $\kappa$ and $T$ are the gas thermal conductivity and temperature. The radiation of the heated gas contributes negligibly to the energy balance, as can be verified by assuming the maximum emission of a black body and finding it to be tiny. At all times, $S_{1}=S_{2}=0$ by mass and momentum conservation, but without approximations, $S_{3} \neq 0$ because the thermal part of the energy density is changed by laser heating and by ionization and recombination of all the relevant species in the gas. However, we recognize that at times $>\sim 10 \mathrm{~ns}$ after laser filament excitation, all of the energy initially stored in free electron thermal energy and in the ionization and excitation distribution of the molecules is repartitioned into a fully recombined gas in its ground electronic state. The "initial" radial pressure distribution driving the gas hydrodynamics at times $>10 \mathrm{~ns}$ is set by the initial conditions $P_{0}(r) \approx\left(f_{e} / f_{g}\right) N_{e}(r) k_{B} T_{e}(r)+$ $2 \Delta \varepsilon_{\text {rot }} / f_{g}$, where $k_{B}$ is Boltzmann's constant, $N_{e}(r)$ and $T_{e}(r)$ are the initial electron density and electron temperature profiles immediately after femtosecond filamentation in the gas, $f_{e}$ and $f_{g}$ are the number of thermodynamic degrees of freedom of the free electrons and gas molecules, and $\Delta \varepsilon_{r o t}$ is the increase in molecular rotational energy density due to the filament. Here, $f_{e}=3$, and $f_{g}=5$ for air at the temperatures of this experiment $\left(\Delta T_{0} \sim 100 \mathrm{~K}\right)$. To simulate the neutral gas response at long time scales, we solve the fluid equations for the $\xi_{i}$, using $S_{3}=0$ and the initial pressure profile given by $P_{0}(r)$ above.

\section{APPENDIX B: OPTICAL SETUP}

A $\lambda=532-n m, 7-n s$ duration beam counterpropagates along a femtosecond filament structure generated by a $10-\mathrm{Hz}$ Ti:Sapphire laser system producing $\lambda=800$-nm, 50 -fs pulses up to $100 \mathrm{~mJ}$. See Fig. 2(a). The optical arrangement is similar to our earlier experiment of Ref. [23]. Here, the 532-nm pulse serves as either a low-energy interferometric probe of the evolving gas-density profile, using a folded wavefront interferometer, or as an injection source for optical guiding in the gas-density structure. The $2 \mathrm{D}$ density profiles were extracted from the interferograms as described in Ref. [23]. The delay of the 532-nm probe/injection pulse is controlled with respect to the Ti:Sapphire pulse with a digital delay generator. The pulse timing jitter of $<10 \mathrm{~ns}$ is negligible given the very-long-time-scale gas evolution we focus on. For the injection experiments, up to $110 \mathrm{~mJ}$ is available at $532 \mathrm{~nm}$. The Rayleigh side-scattering image of Fig. 2(b) was obtained by concatenating multiple images from a low-noise CCD camera translated on a rail parallel to the filament. The images were taken through an 800-nm interference filter.

[1] A. Couairon and A. Mysyrowicz, Femtosecond Filamentation in Transparent Media, Phys. Rep. 441, 47 (2007).

[2] J. K. Wahlstrand, Y.-H. Cheng, Y.-H. Chen, and H. M. Milchberg, Optical Nonlinearity in $\mathrm{Ar}$ and $\mathrm{N}_{2}$ near the Ionization Threshold, Phys. Rev. Lett. 107, 103901 (2011); High Field Optical Nonlinearity and the Kramers-Kronig Relations, Phys. Rev. Lett. 109, 113904 (2012).

[3] J. K. Wahlstrand, Y.-H. Cheng, and H. M. Milchberg, Absolute Measurement of the Transient Optical Nonlinearity in $\mathrm{N}_{2}, \mathrm{O}_{2}, \mathrm{~N}_{2} \mathrm{O}$, and $\mathrm{Ar}$, Phys. Rev. A 85, 043820 (2012); P. Polynkin and M. Kolesik, Critical Power for 
Self-Focusing in the Case of Ultrashort Laser Pulses, Phys. Rev. A 87, 053829 (2013).

[4] Y.-H. Chen, S. Varma, T. M. Antonsen, and H. M. Milchberg, Direct Measurement of the Electron Density of Extended Femtosecond Laser Pulse-Induced Filaments, Phys. Rev. Lett. 105, 215005 (2010).

[5] J. Kasparian, M. Rodriguez, G. Méjean, J. Yu, E. Salmon, H. Wille, R. Bourayou, S. Frey, Y.-B. André, A. Mysyrowicz, R. Sauerbrey, J.-P. Wolf, and L. Wöste, White-Light Filaments for Atmospheric Analysis, Science 301, 61 (2003).

[6] C. D'Amico, A. Houard, M. Franco, B. Prade, and A. Mysyrowicz, Conical Forward $\mathrm{THz}$ Emission from Femtosecond-Laser-Beam Filamentation in Air, Phys. Rev. Lett. 98, 235002 (2007).

[7] K. Y. Kim, A. J. Taylor, J. H. Glownia, and G. Rodriguez, Coherent Control of Terahertz Supercontinuum Generation in Ultrafast Laser-Gas Interactions, Nat. Photonics 2, 605 (2008).

[8] N. Zhavoronkov, Efficient Spectral Conversion and Temporal Compression of Femtosecond Pulses in $\mathrm{SF}_{6}$, Opt. Lett. 36, 529 (2011).

[9] C. P. Hauri, W. Kornelis, F. W. Helbing, A. Heinrich, A. Couairon, A. Mysyrowicz, J. Biegert, and U. Keller, Generation of Intense, Carrier-Envelope Phase-Locked Few-Cycle Laser Pulses through Filamentation, Appl. Phys. B 79, 673 (2004); S. Skupin, G. Stibenz, L. Bergé, F. Lederer, T. Sokollik, M. Schnürer, N. Zhavoronkov, and G. Steinmeyer, Self-Compression by Femtosecond Pulse Filamentation: Experiments Versus Numerical Simulations, Phys. Rev. E 74, 056604 (2006).O. Varela, B. Alonso, I. J. Sola, J. San Román, A. Zaï, C. Méndez, and L. Roso, SelfCompression Controlled by the Chirp of the Input Pulse, Opt. Lett. 35, 3649 (2010).

[10] M. Rodriguez, R. Sauerbrey, H. Wille, L. Wöste, T. Fujii, Y.-B. André, A. Mysyrowicz, L. Klingbeil, K. Rethmeier, W. Kalkner, J. Kasparian, E. Salmon, J. Yu, and J.-P. Wolf, Triggering and Guiding Megavolt Discharges by Use of Laser-Induced Ionized Filaments, Opt. Lett. 27, 772 (2002); R. P. Fischer, A. C. Ting, D. F. Gordon, R. F. Fernsler, G. P. DiComo, and P. Sprangle Conductivity Measurements of Femtosecond Laser-Plasma Filaments, IEEE Trans. Plasma Sci. 35, 1430 (2007); A. Houard, C. D'Amico, Y. Liu, Y. B. Andre, M. Franco, B. Prade, A. Mysyrowicz, E. Salmon, P. Pierlot, and L.-M. Cleon, High Current Permanent Discharges in Air Induced by Femtosecond Laser Filamentation, Appl. Phys. Lett. 90, 171501 (2007).

[11] M. Rodriguez, R. Bourayou, G. Méjean, J. Kasparian, J. Yu, E. Salmon, A. Scholz, B. Stecklum, J. Eislöffel, U. Laux, A. P. Hatzes, R. Sauerbrey, L. Wöste, and J.-P. Wolf, Kilometer-Range Nonlinear Propagation of Femtosecond Laser Pulses, Phys. Rev. E 69, 036607 (2004).

[12] P. Sprangle, J. Peñano, and B. Hafizi, Optimum Wavelength and Power for Efficient Laser Propagation in Various Atmospheric Environments, J. Direct. Energy 2, 71 (2006).

[13] P. Sprangle, J. Peñano, B. Hafizi, D. Gordon, and M. Scully, Remotely Induced Atmospheric Lasing, Appl. Phys. Lett. 98, 211102 (2011).

[14] P. Rairoux, H. Schillinger, S. Niedermeier, M. Rodriguez, F. Ronneberger, R. Sauerbrey, B. Stein, D. Waite, C. Wedekind, H. Wille, L. Wöste, and C. Ziener, Remote Sensing of the Atmosphere Using Ultrashort Laser Pulses, Appl. Phys. B 71, 573 (2000).
[15] J. Liu, J. Dai, S. L. Chin, and X.-C. Zhang, Broadband Terahertz Wave Remote Sensing Using Coherent Manipulation of Fluorescence from Asymmetrically Ionized Gases, Nat. Photonics 4, 627 (2010).

[16] K. W. Fischer, M. R. Witiw, J. A. Baars, and T. R. Oke, Atmospheric Laser Communication: New Challenges for Applied Meteorology, Bull. Am. Meteorol. Soc. 85, 725 (2004).

[17] P. Panagiotopoulos, N. K. Efremidis, D. G. Papazoglou, A. Couairon, and S. Tzortzakis, Tailoring the Filamentation of Intense Femtosecond Laser Pulses with Periodic Lattices, Phys. Rev. A 82, 061803 (2010).

[18] M. Bellec, P. Panagiotopoulos, D. G. Papazoglou, N. K. Efremidis, A. Couairon, and S. Tzortzakis, Observation and Optical Tailoring of Photonic Lattice Filaments, Phys. Rev. Lett. 109, 113905 (2012).

[19] S. Suntsov, D. Abdollahpour, D. G. Papazoglou, P. Panagiotopoulos, A. Couairon, and S. Tzortzakis, Tailoring Femtosecond Laser Pulse Filamentation Using Plasma Photonic Lattices, Appl. Phys. Lett. 103, 021106 (2013).

[20] R. R. Musin, M. N. Shneider, A. M. Zheltikov, and R. B. Miles, Guiding Radar Signals by Arrays of Laser-Induced Filaments: Finite-Difference Analysis, Appl. Opt. 46, 5593 (2007).

[21] M. Châteauneuf, S. Payeur, J. Dubois, and J.-C. Kieffer, Microwave Guiding in Air by a Cylindrical Filament Array Waveguide, Appl. Phys. Lett. 92, 091104 (2008).

[22] S.-B. Wen, C.-F. Chen, X. Mao, and R. E. Russo, Guiding and Focusing of a Nanosecond Infrared Laser within Transient Hollow Plasma Femtosecond Filament Channels, J. Phys. D 45, 355203 (2012).

[23] Y.-H. Cheng, J. K. Wahlstrand, N. Jhajj, and H. M. Milchberg, The Effect of Long Timescale Gas Dynamics on Femtosecond Filamentation, Opt. Express 21, 4740 (2013).

[24] K. Y. Kim, I. Alexeev, and H. M. Milchberg, Single-Shot Measurement of Laser-Induced Double Step Ionization of Helium, Opt. Express 10, 1563 (2002).

[25] L. Levi, O. Lahav, R. A. Nemirovsky, J. Nemirovsky, I. Orr, I. Kaminer, M. Segev, and O. Cohen, Long-Lived Waveguides and Sound Wave Generation by Laser Filamentation, arXiv:1307.3588.

[26] J. K. Wahlstrand, N. Jhajj, E. W. Rosenthal, S. Zahedpour, and H. M. Milchberg, Direct Imaging of the Acoustic Waves Generated by Femtosecond Filaments in Air, Opt. Lett. 39, 1290 (2014).

[27] See Supplemental Material at http://link.aps.org/ supplemental/10.1103/PhysRevX.4.011027 for movies showing the time-dependent gas density dynamics and the guiding of light in the thermal waveguide.

[28] A. W. Snyder and J. D. Love, Optical Waveguide Theory (Chapman and Hall, London, 1991).

[29] K. P. Birch, Precise Determination of Refractometric Parameters for Atmospheric Gases, J. Opt. Soc. Am. A 8, 647 (1991).

[30] See http://webbook.nist.gov/chemistry/fluid/.

[31] M. D. Feit and J. Fleck, Light Propagation in Graded-Index Optical Fibers, Appl. Opt. 17, 3990 (1978).

[32] V. V. Vorob'ev, Thermal Blooming of Laser Beams in the Atmosphere, Prog. Quantum Electron. 15, 1 (1991). 